\title{
Design Concept Study for Cerenkov Monitoring Devices Based Upon CCD Technology
}

\author{
J. K. Partin
}

July 1997

Idaho National Engineering and Environmental Laboratory Lockheed Martin Idaho Technologies Company Idaho Falls, Idaho 83415

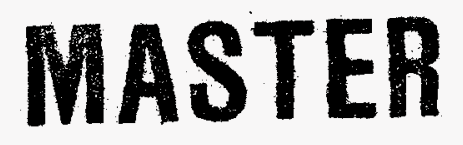

Prepared for the

U. S. Department of Energy

Office of Nonproliferation and National Security

Under DOE Idaho Operations Office

Contract Number DE-AC07-94ID13223 


\section{DISCLAIMER}

This report was prepared as an account of work sponsored by an agency of the United States Government. Neither the United States Government nor any agency thereof, nor any of their employees, make any warranty, express or implied, or assumes any legal liabii. ty or responsibility for the accuracy, completeness, or usefulness of any information, apparatus, product, or process disclosed, or represents that its use would not infringe privately owned rights. Reference herein to any specific commercial product, process, or service by trade name, trademark, manufacturer, or otherwise does not necessarily constitute or imply its endorsement, recommendation, or favoring by the United States Government or any agency thereof. The views and opinions of authors expressed herein do not necessarily state or reflect those of the United States Government or any agency thereof. 


\section{DISCLAIMER}

Portions of this document may be illegible electronic image products. Images are produced from the best available original document. 


\begin{abstract}
A design concept study for the development of Cerenkov monitoring devices based upon Charge-Coupled Device (CCD) technology is presented. The study provides a description of the technology and the features which characterize the performance of specific devices. Capabilities of commercially-available CCD systems are reviewed and and tradeoffs between the various system attributes are examined for two different monitoring scenarios: one in which the device is designed to be transportable for use by a visiting inspector; and one in which the device is mounted in the ceiling, or other fixed position, and the data is transmitted to off-site locations for evaluation.
\end{abstract}




\section{Design Concept Study for Cerenkov Monitoring Devices Based Upon CCD Technology}

\section{INTRODUCTION}

Accurate tracking of the international spent fuel inventory is an increasingly important task, in view of the new political and social pressures within the world community. These inventories are regularly audited by the International Atomic Energy Agency (IAEA) inspectors to minimize the possibility that nuclear materials are diverted for other purposes. These audits compare the declared inventory against the number of fuel assemblies on the site; and in addition, determine the authenticity of some of the fuel assemblies. Historically, a combination of techniques have been used for verification including visual observation, nuclear counting and video surveillance.

In recent years, the detection of Cerenkov radiation from the irradiated fuel has become an attractive means of determining the approximate radiation history of the stored fuel (1-2). Cerenkov radiation in fuel storage pools arises from the passage of fast $(>0.26 \mathrm{MeV})$ electrons through the water. The fast electrons, in turn, are produced by the decay of fission products in the fuel elements. Production mechanisms include Compton scattering of gamma rays in water, beta decay and pair production. The intensity of the blue Cerenkov light is therefore directly related to the fission product inventory of the spent fuel element and serves as a measure of its radiation history (3).

Early detection devices were based upon image intensifier tubes used in night vision instrumentation. These types of devices exhibited broadband spectral responses that required the pool area to be darkened during the viewing. To allow operation with lighting, devices were then equipped with filters to reduce interferences from facility illumination. This allowed the detection of Cerenkov, but often reduced the image contrast to the extent that the details of the assemblies could not be resolved.

Charge-Coupled Device (CCD) technology is now available which would greatly improve the monitoring of Cerenkov radiation for fuel inspections. This instrumentation offers both improved spatial resolution and sensitivity over intensified tube technology. Devices can be configured for deployment as either portable on-site tools or fixed position continuous monitoring instruments. Systems can have up to 22-bit dynamic range allowing them to detect very small intensity changes such as those expected when a single rod is removed from a densely packed assembly. The digital CCD output is also easy to manipulate, analyze and archive using standard computer software. 
Systems to be used for the inspection of fuel inventories should meet the following general requirements:

(1) The system should allow the inspector to examine the fuel assembly in enough detail to identify any missing or "dummy" fuel rods.

(2) The system should allow the inspector to make an accurate estimation of the radiation history of the fuel within the assembly, with sufficient sensitivity to monitor fuel that has been in storage for a minimum of ten years.

(3) The system should be noninvasive and not interfere with the normal operation of the facility.

(4) The system should allow fuel assemblies to be examined at a rate of 10 seconds per assembly.

(5) The system should provide a means of archiving data.

(6) The system should be portable, self-contained, easy to align and use, and relatively inexpensive to acquire and maintain.

This study will evaluate the degree to which Cerenkov monitoring using CCD technology can meet these requirements. Capabilities of commercially available CCD systems are reviewed and trade-offs between various system features for this application will be examined. 


\section{CCD CAMERA DESCRIPTION AND PERFORMANCE}

\section{CCD Camera Description}

A charge-coupled device (CCD), illustrated schematically in Figure 1, is a two dimensional array of photosensors. The CCD architecture is designed for three basic functions: charge collection, charge transfer, and the conversion of charge into a measurable voltage. The basic building block of the device is the metal oxide semi-conductor capacitor which serves as a gate. By manipulating the voltages of these gates, charge can either be stored or transferred. The gate is produced by growing a thin layer of silicon dioxide on a section of silicon and then applying a transparent conductive layer. When a positive potential is applied to the gate, a depletion region is created where photon-generated charge may be stored. (Thermal energy and high energy particles may also generate photons which are indistinguishable from those generated by photons, and constitute sources of noise in the system).

Imagers are typically processed on silicon wafers that are 10 to 20 centimeters in diameter and 500 microns thick. A matrix arrangement of oxide and gate structures can be produced so that many thousands of potential wells are established across a large area of silicon. The geometry of the buried channel establishes the column boundaries and prevents lateral charge migration. Along the length of the columns, the charge is contained in individual wells by potentials that are applied to a multiphase gate structure. By this means, it is possible to support a two-dimensional array of independent potential wells.

The imaging device illustrated in Figure 1 has 262,144 wells, called pixels, each of which is capable of storing photon-generated electronic charge. The two-dimensional array of pixels is called the parallel register. An image focused on this register produces a pattern of charge in proportion to the total integrated photon flux incident on each pixel. The array can be programmed to collect photonic charge over a prescribed period of time. The total charge collected is then equal to the product of the photonic charge generation rate and the exposure time.

After an exposure, a programmed sequence of gate potentials causes all the charge packets stored in the parallel register to be shifted up one row, towards the serial register. The charge stored in the top row is shifted from the parallel register into the serial register. From the serial register, charge packets are individually shifted toward the output amplifier. The amplifier produces a signal that is proportional to the quantity of charge in each charge packet. When the serial register is emptied of charge, a second row of charge packets is shifted in the from the parallel register for transport to the output amplifier. As the readout process continues empty rows are left at the bottom of the parallel register. When the entire register has been emptied, a new exposure is made and the process is continued. 


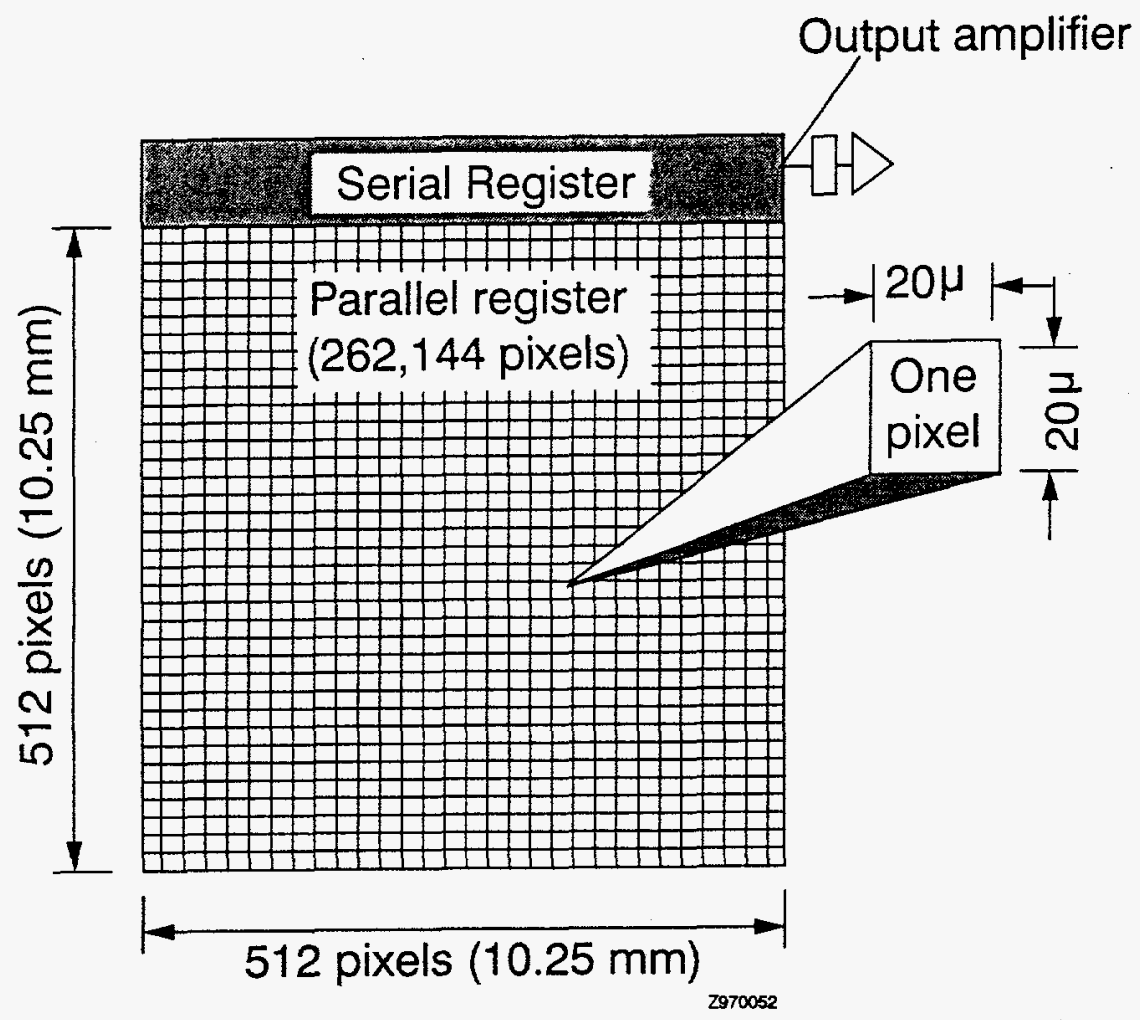

Figure 1. Typical Scientific-Grade CCD Imager (4).

With many arrays, it is also possible to shift more than one row of charge into the serial register. Similarly, it is possible to shift more than one serial register element into a summing gate just before the output node. This is referred to as charge grouping, binning, or super pixeling. Binning increases the signal output and dynamic range at the expense of spatial resolution. Because it increases the signal-to-noise ratio, binning is often used in low light applications where resolution is less important.

Several array architectures for electronic imaging are also available. The full frame CCD architecture is the simplest. This device employs a single parallel register for photon exposure, charge integration, and transport. A shutter is used to control the exposure and to block light from striking the CCD during the read process which would otherwise result in charge smearing. The frame transfer $\mathrm{CCD}$ has a parallel register that consists of two nearly identical arrays, one for imaging and one for storage. The storage cells are identical in structure to the light sensitive cells but are covered with a metal light shield to prevent any light exposure. After the integration cycle, the charge is quickly transferred from the light sensitive pixels to the storage cells. A frame transfer CCD can operate continuously, without an shutter, at television rates. The interline transfer $\mathrm{CCD}$ has a parallel register that has been subdivided into stripes so that the opaque storage register fits between columns of pixels. The electronic image accumulates in the exposed area of the parallel register as it does in the frame transfer CCD. During CCD readout, the entire image is shifted under the interline mask into a hidden shift register. The main advantage with 
interline transfer is that the process is rapid enough that there is no need to shutter the incoming light. These types of CCDs exhibit poor sensitivity since a large portion of each pixel is covered by the opaque strip mask, and therefore, they are more commonly used in customer electronics and not for scientific application.

\section{CCD Camera Performance}

CCD cameras are generally characterized by a number of features including: format and resolution, spectral response or quantum efficiency, dark charge, well capacity, dynamic range, read-out noise, and scan rate.

In considering the appropriate format and resolution the important parameters are pixel size, pixel density, aspect ratio and overall package size. The resolution of a CCD camera is determined by the geometry of the CCD. Cameras are generally described in terms of resolution elements, or pixels, which are usually square in geometry and may vary in size from 6 to 30 microns. Typical formats may vary from 576 X 384 pixel array for a spectroscopic application to $4096 \mathrm{X} 4096$ for detailed scientific imaging applications. An array of $576 \times 384$ pixels is a common format for TV application. The system cost generally scales with resolution.

The spectral response or quantum efficiency of a $\mathrm{CCD}$ is the measure of the effectiveness of the imager to produce electronic charge from incident photons of a particular energy or wavelength. In an ideal material, when the photon energy is greater than the semiconductor band gap energy (1080 nanometers for silicon), each photon incident upon the material will produce on electron-hole pair for a quantum efficiency of one. However, the absorption of photons in silicon is wavelength dependent, decreasing at longer wavelengths. This means that longer wavelength photons are absorbed deeper into the substrate than short wavelengths. And very long wavelength photons may pass through the CCD without being absorbed. The polysilicon electrode structures also reflect nearly $60 \%$ of the impinging near-infrared radiation; and in addition, absorb nearly all of the radiation at wavelengths of 400 nanometers or less.

The blue region response can be increased by using thinner polysilicon gates, or by depositing a ultraviolet phosphor onto to the array, as illustrated in Figure 2. The light emitting phosphor, often referred to a downconverter, absorbs light at short wavelengths (200-400 nanometers) and re-emits light at a lower energy, typically in the visible region of the spectrum. The downconverter is completely transparent to longer wavelengths and therefore does not degrade the quantum efficiency in the visible region. Phosphors radiate in all directions and only the light fluorescing toward the array is absorbed. An effective quantum efficiency of about $15 \%$ is typical for these types of devices. 


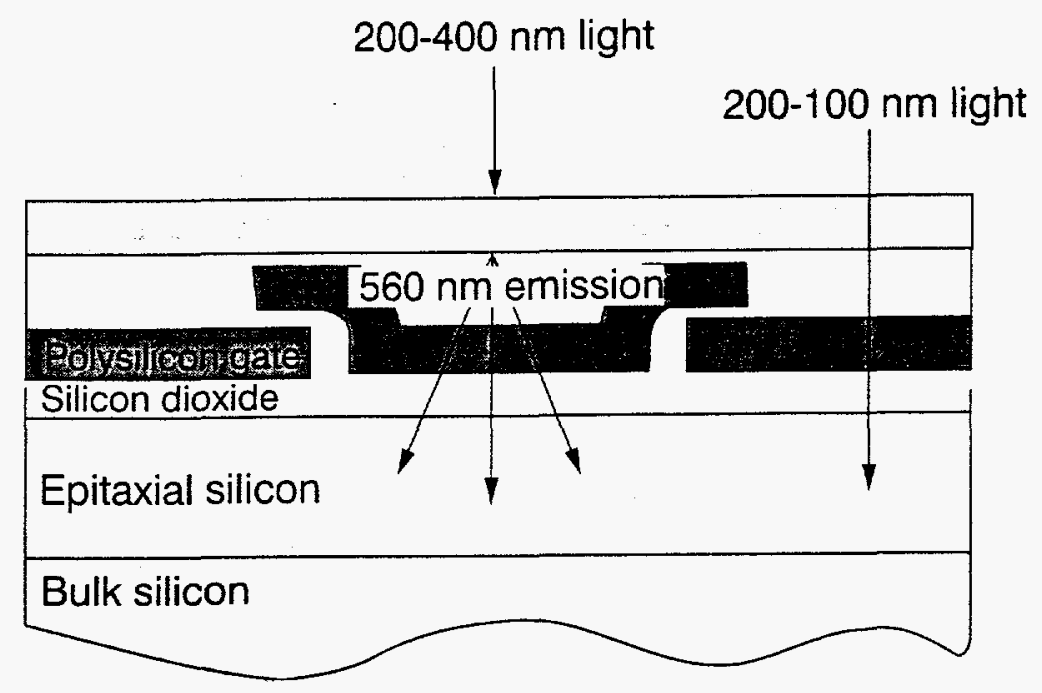

Figure 2. Front-Illuminated CCD with Phosphor Coating (4).

The optimal spectral response (highest quantum efficiency) is achieved by thinning the device and focusing the image on the backside as shown in Figure 3. Illuminating the array from the back avoids the polysilicon gate problem. The material is thinned in order to allow short wavelength radiation to produce electron-hole pairs in region where the generated charge can reach a storage site. (Short wavelength photons tend to be absorbed near the surface of the material, and therefore, the electron-hole pairs recombine before reaching a storage site.) With the application of the proper anti-reflection coating, these devices can achieve quantum efficiencies of $85 \%$. However, these devices are fragile, complex and more expensive than phosphor-coated devices.

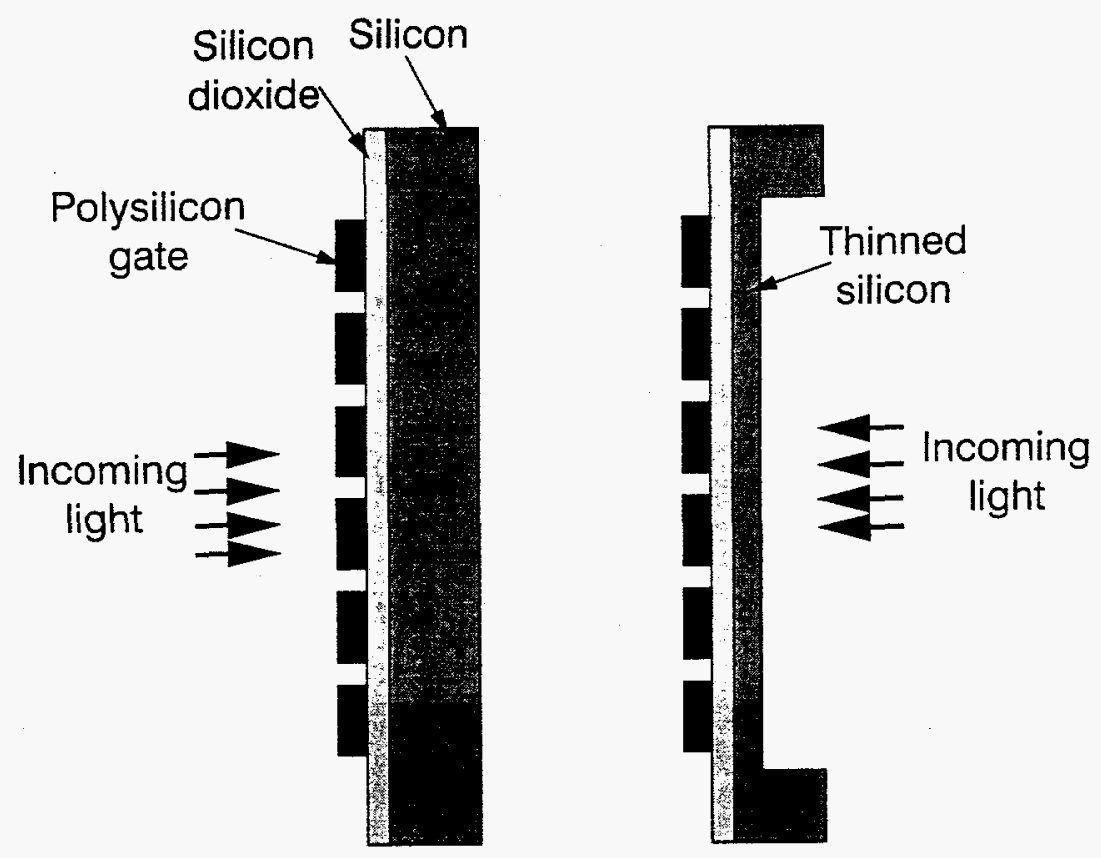

Figure 3. Front and Backside Illuminated CCDs (4). 
The quantum efficiency of the various CCD configurations is summarized in Figure 4.

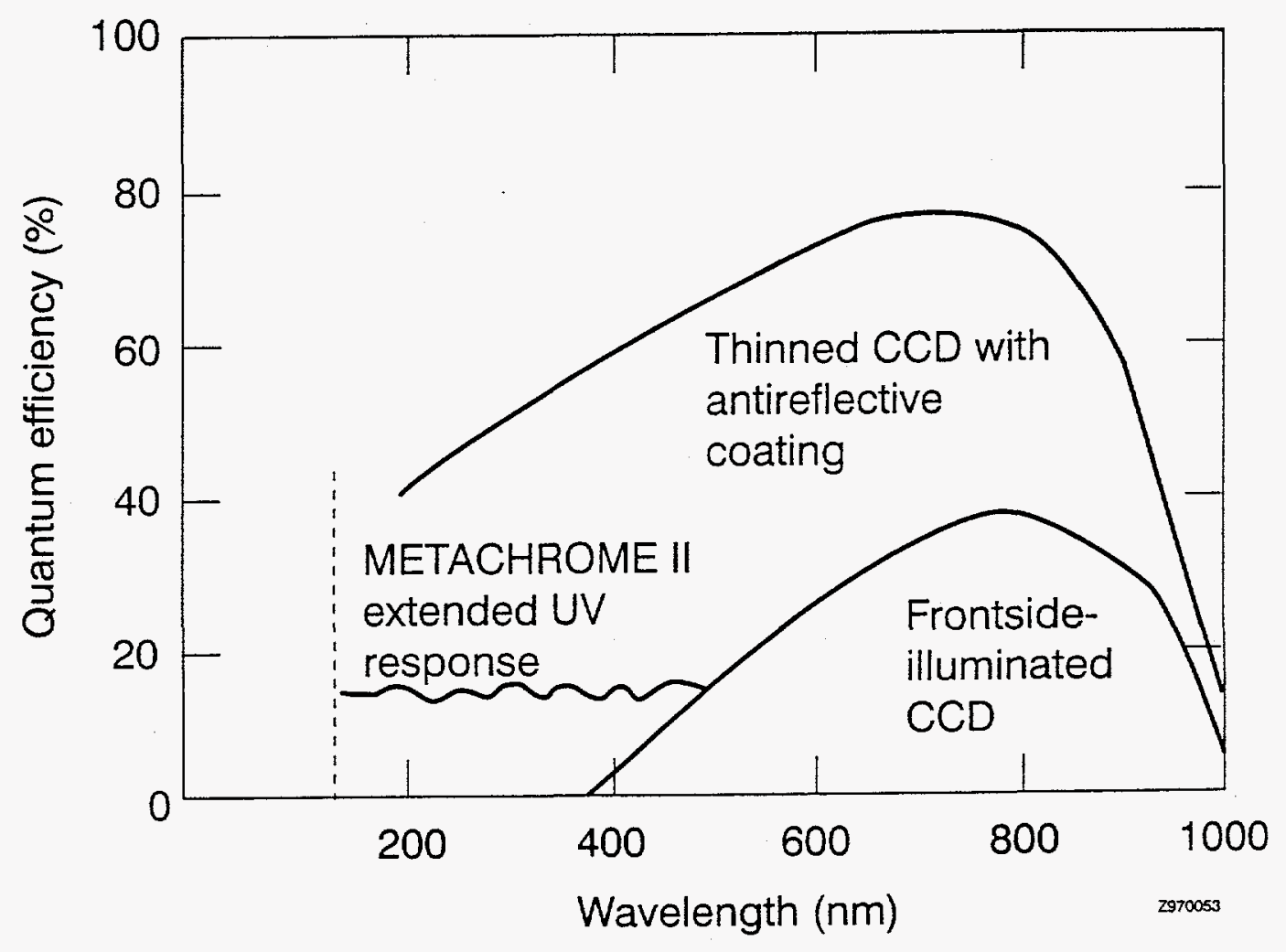

Figure 4. Quantum Efficiencies of CCDs (4).

Dark current or dark charge are terms which refer to the signal produced by thermallygenerated charges. Dark current is generated in both the bulk of the silicon, and at the surface of the silicon in the silicon to silicon-oxide interface regions. This charge is indistinguishable from the signal-generated charge and constitutes a source of noise in the system. Dark current densities vary significantly among CCD manufacturers with values ranging from 0.1 nanoamperes $/ \mathrm{cm}^{2}$ to 10 nanoamperes $/ \mathrm{cm}^{2}$. Dark current increases the background level, reducing the dynamic range and increasing the overall system noise. Unlike background signals, it cannot be reduced or eliminated by digital substraction.

In principle, the dark current density can be made negligible by cooling, decreasing a factor of two for every $7-8^{\circ} \mathrm{C}$ in array temperature. The most commonly used cooling scheme is a thermoelectric (TE) cooler. TE coolers are Peltier devices driven by an electric current that pumps heat away from the CCD to a heat sink. The heat sink is then cooled by passive air, forced air or forced liquid (usually water). The final array temperature depends upon the amount of heat generated in the array, the cooling capacity of TE device, and the temperature of the TE heat sink. Peltier devices can cool down to about $-60^{\circ} \mathrm{C}$. TE coolers can be integrated into the same package with the array.

For long integration periods, especially when pixels are binned or added together, cryrogenic cooling using liquid nitrogen as a coolant is recommended. Although liquid nitrogen 
is at $-200^{\circ} \mathrm{C}$, the typical operating temperature is -60 to $120^{\circ} \mathrm{C}$. Condensation is a problem and the arrays must be placed in an evacuated chamber, or one filled with a dry atmosphere.

New CCDs are also now available in which the generation of dark current is reduced by a factor 20-500 fold, depending upon the characteristics of the device and the particular design. This level of reduction greatly reduces the CCD cooling requirements, allowing room temperature operation for some applications. These devices are based upon multipinned phased (MPP) technology. MPP CCDs have special implants under the electrodes and utilize a different phase clocking scheme. Consequently, surface charge is not collected during integration. Because most of the dark charge is produced at the surface, the overall dark charge is reduced dramatically. The downside of this technology is that the charge wells in these devices are 2-3 times smaller than conventional devices and these devices are the most likely to saturate.

Well capacity is defined as the maximum number of electrons that can be contained in a single potential well without spilling over to adjacent regions (blooming). There are three different well capacities that need to be considered in the design of an imaging device: the value for the imaging section, the value for the horizontal shift register, and the value representing the preamp node capacitance. The first value relates to the well capacity of an individual pixel in the light sensitive (imaging) detection region. CCD arrays with a pixel size on the order $20 \mu \mathrm{m} \times 20 \mu \mathrm{m}$ have typical well capacities of 400,000-750,000 electrons. For an MPP device, this is reduced to around 120,000-250,000 electrons. Devices with smaller pixels $(10 \mu \mathrm{m} \times 10 \mu \mathrm{m})$ have smaller capacities, i.e. $40,000-80,000$ electrons. The imaging well capacity normally sets the dynamic range.

The dynamic range of a $\mathrm{CCD}$ is defined as the ratio of the full well saturation charge to the system noise level, expressed in electrons. The system noise may vary between a few electrons in a liquid nitrogen-cooled scientific camera to many thousands of electrons in an inexpensive video camera. As described in the previous description of well capacity, saturation charge is dependent upon the well capacity and of the CCD and varies with device architecture and pixel size. Saturation charge scales more or less linearly with pixel area and is independent of the system noise level.

Readout noise originates in the output preamplifier of the CCD. This preamp measures very small variations in the voltage produced on a small node capacitor each time the charge content of one or more pixels is transferred to it by the shift register. Readout noise is independent of the dark current and signal levels, but is dependent upon the temperature and the readout rate. (The rate of increase depends upon the electrical design of the CCD and its associated electronics.) Readout noise generally sets the detection limit of a CCD. 
As an example, the horizontal shift register has a typical well capacity of $250,000-900,000$ electrons. When binning is performed along the column and therefore in the shift register, the well capacity of the shift register sets the overall limit on the dynamic range. For example, if the readout noise is 10 electrons RMS and the well capacity is 320,000 electrons then the maximum dynamic range is $320,000 / 10$ or 32,000 or about 15 bits. The preamp node capacitance is the most important in a binning mode, since it has to read the charge inte-grated over the entire binned region.

CCD arrays can be read out at various rates depending upon the design of the CCD and the external associated electronics. Scan rates vary from "slow", $50 \mathrm{kHz}$, to up to several $\mathrm{MHz}$. Operation at slow scan rates substantially reduces the readout noise and is generally employed for low flux applications requiring very high sensitivity. 


\section{EVALUATION OF CCD FEATURES FOR CERENKOV MONITORING APPLICATONS}

\section{Factors Affecting Cerenkov Monitoring}

In addition to fuel type and radiation history, there are a number of factors which affect the intensity of Cerenkov light emitted by a fuel assembly, and therefore, complicate the interpretation of data from monitoring systems. For example, the fuel assembly design may block a considerable fraction of the emitted Cerenkov photons. The presence of a "hot" element may produce radiation that appears to come from cooler neighboring assemblies. The radiation is extremely collimated, and consequently very susceptible to changes in the viewing angle. The water depth and clarity are also factors. As illustrated in Figure 5, Cerenkov is strongly absorbed in water at wavelengths less than 350 nanometers and greater than 650 nanometers (5). Ordinarily this observation would suggest that the monitoring system should be designed to operate in the wavelength range of 400 to 600 nanometers; however, as illustrated in Figure 6, there is the possibility of interference from the facility illumination, since commonly used light sources also emit in this region.

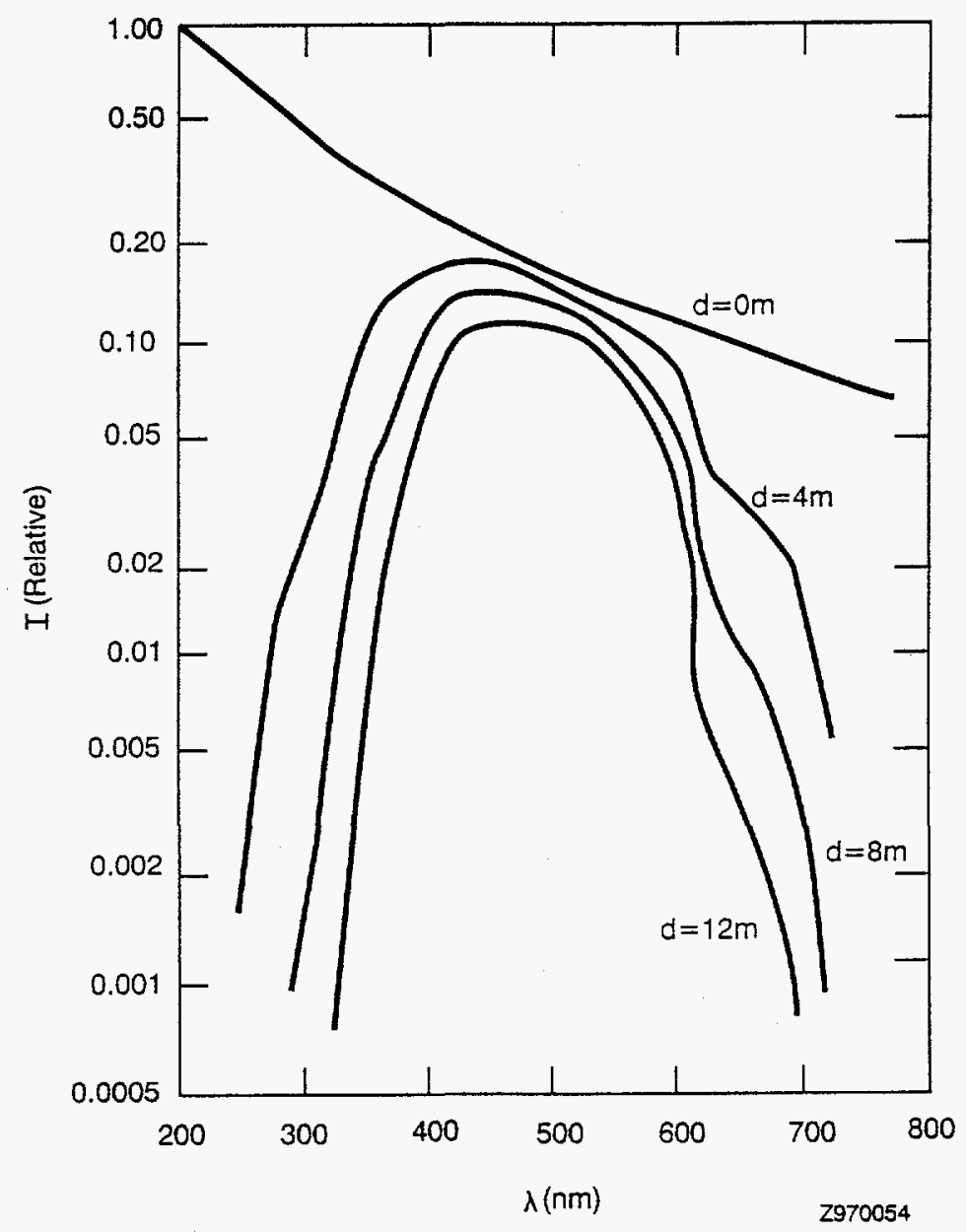

Figure 5. Cerenkov Intensity as a Function of Wavelength for Various Water Depths (5). 

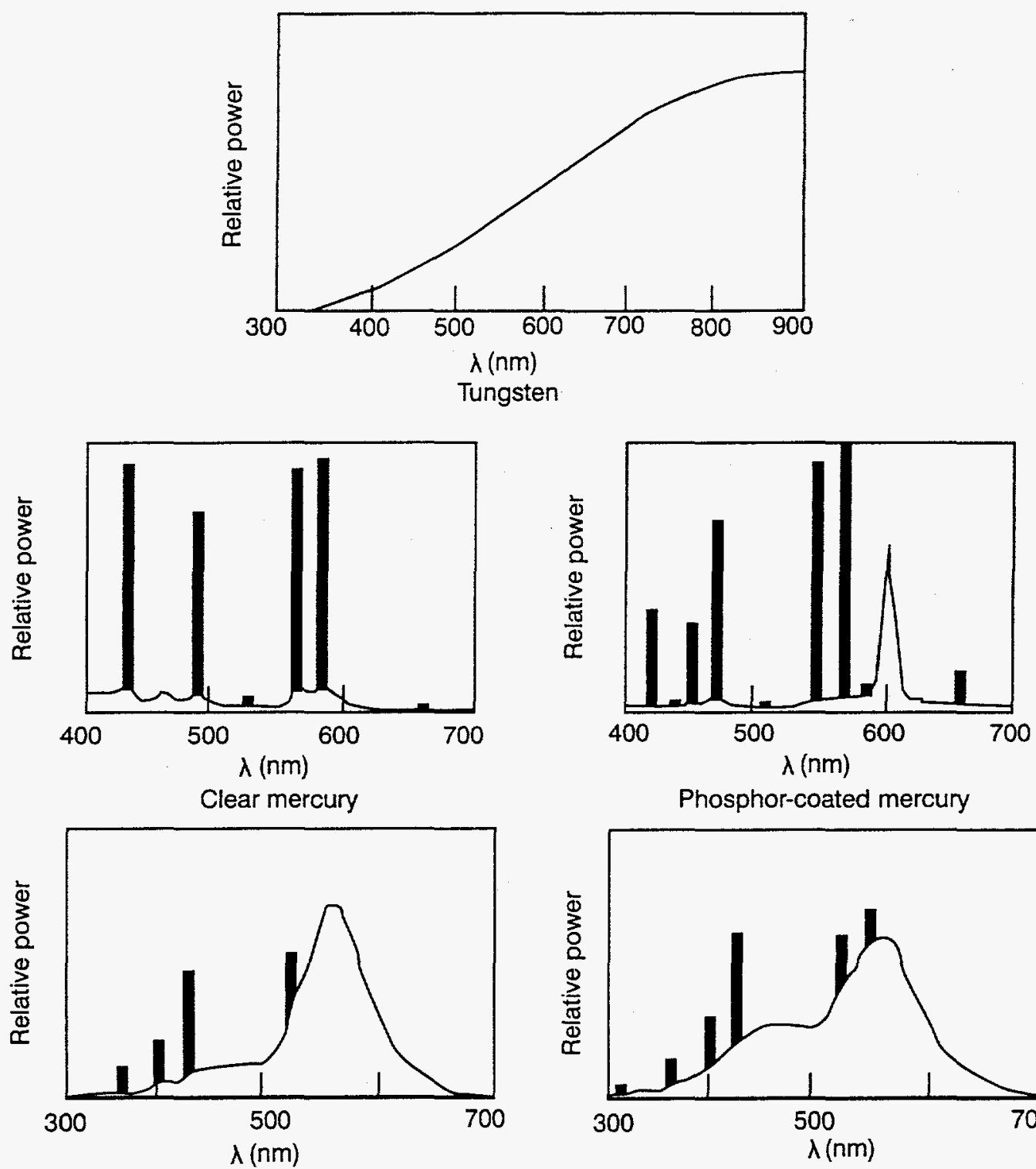

Phosphor-coated mercury

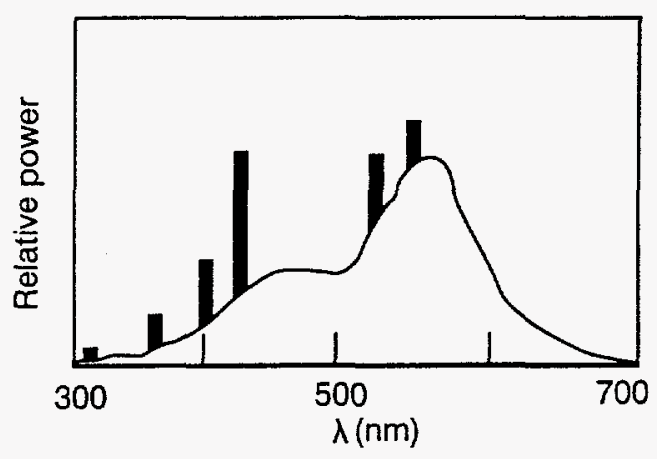

Warm white fluorescent

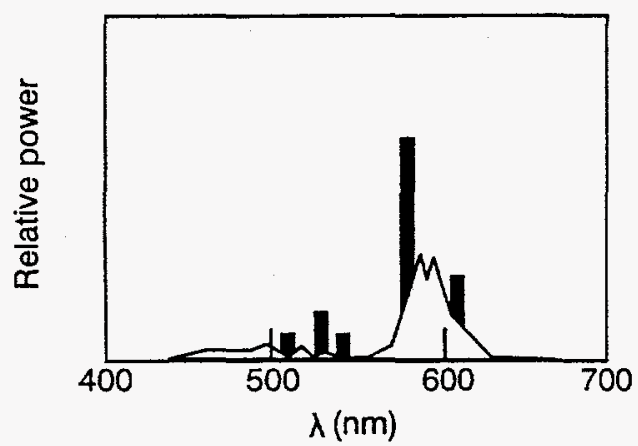

High pressure sodium

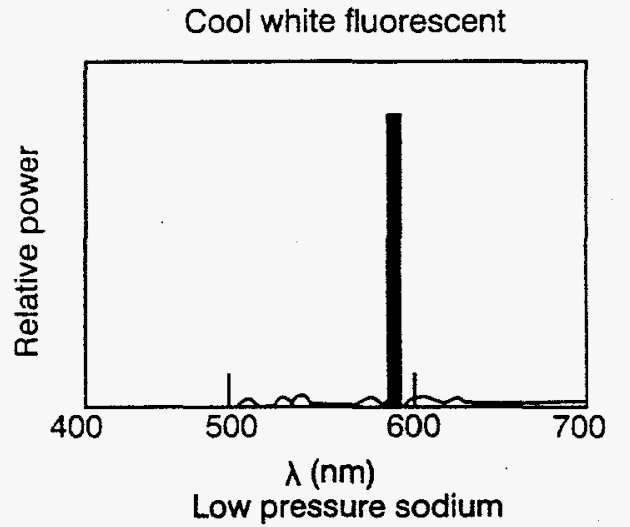

Figure 6. Spectral Distribution of Various Light Sources (5). 


\section{Deployment Scenarios}

There are two differing deployment scenarios for verifying spent nuclear fuel inventories. The first, which is currently in use, involves the examination by on-site inspectors. In this scenario, the inspector visually inspects the fuel assembly with the aid of a portable monitoring device and makes a determination based upon readings from the device and his or her previous experience. For the examination, the monitoring system is generally mounted upon the canal bridge structure and the bridge is moved to allow the viewing of different assemblies. This type of examination requires that the monitoring of a single assembly be completed as quickly as possible, 10 seconds is the desired throughput rate.

The second scenario involves the permanent installation of a monitoring device in the facility, as shown schematically in Figure 7. This type of monitoring, although politically sensitive, could relax the technical requirements for the system compared to those necessary for a quick look inspection tool, allowing both more thorough and inexpensive verifications of spent fuel. Devices for both deployment scenarios are considered in this section.
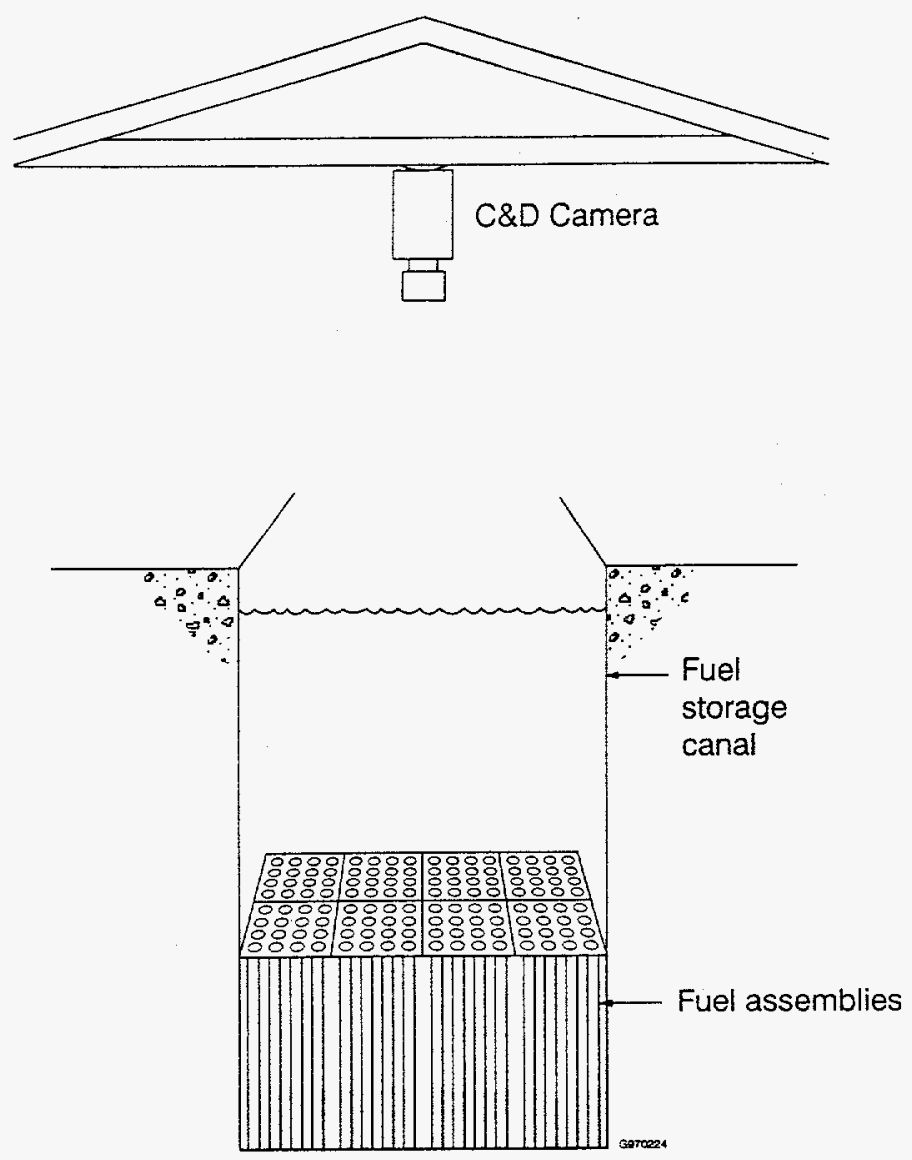

Figure 7. CCD Device Installed at Storage Facility. 


\section{CCD Design Features for Portable and Fixed Position Monitoring Devices}

The monitoring devices are required to resolve the fuel elements in various types of fuel assemblies, as illustrated in Figure 8. A general rule of thumb requires that the object be projected over at least three pixels for good signal-to-noise detection, and of course the detail in the image will improve as more pixels are used. A fuel element is approximately 10 millimeters in diameter. Assuming the detector pixel is 20 microns square, the required

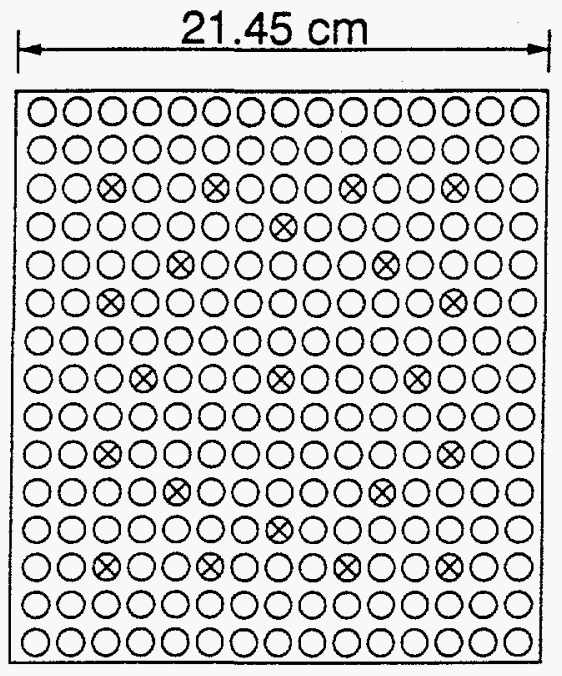

Fuel rod positions

$\otimes$ Fuel tube positions

\section{Pressurized water reactor}

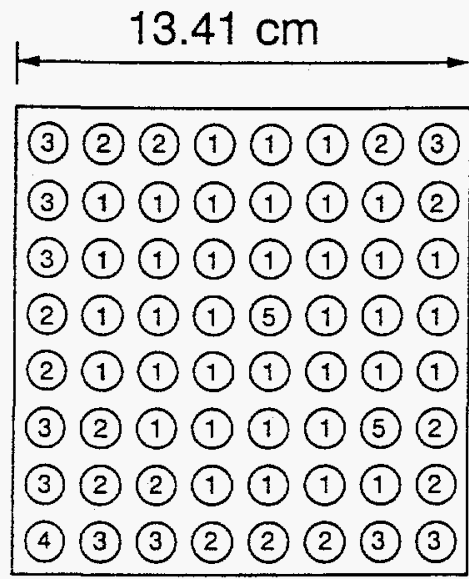

Boiling water reactor

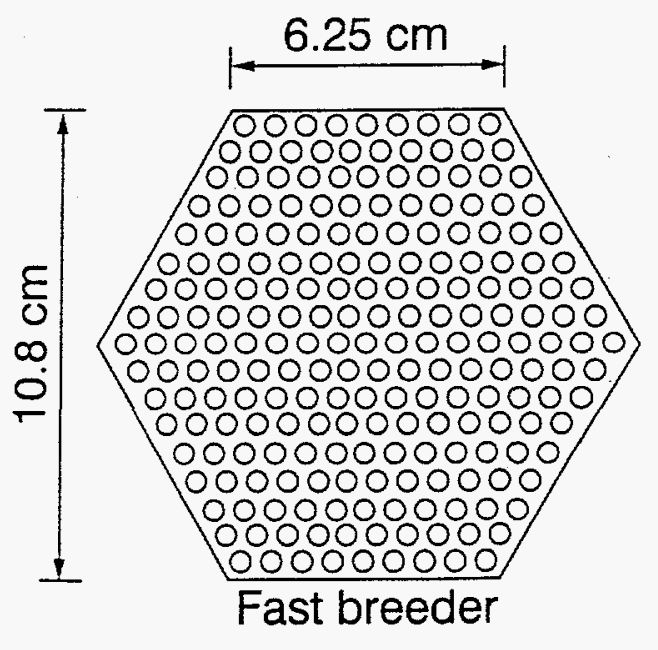

G970e25

Fuel assembly cross sections

Figure 8. Fuel Assembly Cross Sections. 
magnification for a system which will just resolve a fuel element can be calculated as follows:

$$
\mathbf{M}=\frac{\text { Image Size }}{\text { Object Size }}=\frac{3 \times 20 \times 10^{-6} \mathrm{~m}}{10 \times 10^{-3} \mathrm{~m}}=0.006
$$

A typical fuel assembly is located approximately 12 meters below the bridge structure where the monitoring device is mounted for the inspection. Using this distance as the object distance, $\mathrm{R}$, the effective focal length (efl) of the lens to image the assembly can be calculated,

efl $=\mathrm{RM}=12 \times 0.006 \mathrm{~m}=75 \mathrm{~mm}$

The array size to image a single fuel assembly is

$22 \mathrm{~cm}\left(10^{4} \frac{\mu \mathrm{m}}{\mathrm{cm}}\right) X(0.006)=\frac{1320 \mu \mathrm{m}}{20 \mu \mathrm{m}}=66$ pixels

This implies that with a standard size CCD camera $(512 \times 512)$, an $8 \times 8$ array of fuel assemblies could be imaged and the fuel elements within these assemblies could be resolved. In order to obtain more detail in an assembly the image could be magnified, projecting the fuel element over 10 pixels. This would result in a magnification of 0.020 , and consequently, 220 pixels would be required to image one assembly and a standard size camera could only observe a $2 \times 2$ array of assemblies.

The fixed position, ceiling mounted device would have a similar format; however, in this case the effective focal length would of course be longer due to the increased object distance. Assuming a distance of $25 \mathrm{~m}$, the efl is now,

efl $=25 \times 0.006 \mathrm{~m}=150 \mathrm{~mm}$

In this case, it would be desirable to image as many fuel assemblies as practical. Using a 1024 X 1024 format camera, this system could examine a $16 \times 16$ matrix of fuel assemblies.

Other important design criteria for Cerenkov monitoring involve establishing the required detectability, sensitivity, and dynamic range of the device. Our calculations of the total photon flux emitted from the Advanced Test Reactor fuel indicate that on the order of $10^{15}$ Cerenkov photons are emitted from the these assemblies. Of these photons many are scattered and absorbed by the infrastructure of the storage rack and the fuel assemblies, others are not transmitted through the water and/or collected by the aperture of the optical system. The number of emitted photons also of course depends upon the radiation history of the fuel. The number of photons which could be expected to be detected in a 
meter by meter plane above the stored fuel could vary between 100 's of millions to a few hundred thousand (7).

The detectability refers to the signal whose magnitude is just equal to that of the noise fluctuations. The overall noise, $\mathrm{N}_{\mathrm{T}}$, associated with the acquisition of an image by the $\mathrm{CCD}$ is defined as:

$N_{T}=\left[N_{R}^{2}+N_{D}^{2}+N_{p h}^{2}\right]^{1 / 2}$ where,

$\mathrm{N}_{\mathrm{R}}=$ Readout noise of the CCD

$N_{D}=$ Dark charge noise of the CCD

$\mathrm{N}_{\mathrm{ph}}=$ Photon shot noise associated with the signal.

The signal-to-noise $(\mathrm{S} / \mathrm{N})$ defines the precision with which the measurement can be made. The dynamic range determines the maximum signal the CCD can detect and establishes the resolution of the measurement.

For application as a portable inspection device, the following $\mathrm{CCD}$ features are desirable: a standard CCD format of 512 X 512 pixels with TE forced air cooling, operating in the frame transfer mode. The use of the standard size reduces cost, while maintaining reasonable viewing resolution. The TE forced air cooling allows operation at a temperature of around $-40^{\circ} \mathrm{C}$ without having to obtain access to water or liquid nitrogen; and in addition, keeps the dark current at a reasonably low rate (around 1 electron/pixelsecond). The selection of the frame transfer mode allows near real-time viewing of the acquired image.

This system is required to detect photon fluxes which may vary from a few photons/second-pixel to a few thousand photons/second-pixel. In order to avoid disturbing the operation of the facility, the device must be operable under normal illumination conditions. This generally requires the filtering of spectral wavelengths greater than 350 nanometers. The need to operate at short wavelengths requires the use of a phosphor coating on the array to obtain a reasonable quantum efficiency (see figure 4). Subtle differences in intensities must be detectable in order to recognize the replacement of individual fuel elements within dense assemblies. These differences may be on the order of few percent requiring a high precision, high resolution measurement capability. The measurement should be performed at a rate of 10 seconds per fuel assembly. Using the previously calculated view of $8 \times 8$ matrix of assemblies, an integration of time up to 600 seconds could be used and still meet this criteria.

The expected performance of the portable system is determined as follows:

At low levels of illumination, the incoming signal flux is on the order of 5 photons/second-pixel. The quantum efficiency at 300 nanometers is 0.15 for an ultraviolet enhanced system. The dark current for a TE-cooled system is on the order of 1 electron/pixel-second, and the readout noise is around 20 electrons at $5 \mathrm{MHz}$. 
The system performance, using an integration time of 600 seconds, can be evaluated with the following relationships,

$\mathrm{N}_{\mathrm{R}}=20$ electrons

$N_{D}=(1 \text { electron/second-pixel X } 600 \text { seconds })^{1 / 2}=25$ electrons

$\mathrm{N}_{\mathrm{ph}}=(5 \text { photons/second-pixel } \times 0.15 \times 600 \text { seconds })^{1 / 2}=21$ electrons

$\mathrm{N}_{\mathrm{T}}=\left[20^{2}+25^{2}+21^{2}\right]^{1 / 2}=38$ electrons

$\mathrm{S} / \mathrm{N}=\frac{\text { Integrated Photon Flux }}{\text { Noise }\left(\mathrm{N}_{\mathrm{T}}\right)}=\frac{450}{38}=11.8 @ 8.5 \%$ precision

The detectability of the system is

Detectability $=\frac{\text { Noise }}{\mathrm{QE}}=\frac{38}{0.15}=253$ photons

The dynamic range of the system is calculated from the well capacity, which is on the order of 200,000 electrons for a frame transfer CCD system, and the baseline noise.

Dynamic Range $=\frac{200000}{38}=5263$ or around 12 bits

While this system will produce an acceptable performance for many inspection activities, several potential problems should be pointed out. First, three pixel resolution of the fuel elements may not be adequate to unambiguously detect fuel assemblies in all storage configurations. Second, an integration time of 10 minutes may be unrealistic for a bridgemounted device. In practice the bridge structure may be subject to vibrations from pumps circulating the water in the storage canal, or other operational perturbations. The effective impact of fixing both of these problems is a decrease in integration time and/or system throughput that would greatly reduce the performance of the device. (For example, substituting a 60 second integration time into the above equations results in the measurement precision degrading from nominally $8 \%$ to $50 \%$.)

There are also several ways of improving the performance of the device, albeit at increased cost or inconvenience. As illustrated in figure 4 , the quantum efficiency could be improved by using a back-illuminated chip configuration. Increasing the quantum efficiency could improve the $\mathrm{S} / \mathrm{N}$ by a factor of 10 , but significantly increases the cost of the instrument. Noting from the noise calculation that a significant component to the noise is introduced in readout and dark currents, increases in performance can be effected by cooling the system to a lower temperature by using water circulation or by using a slower readout rate. The first fix is inconvenient to implement in the field, and the second reduces the fuel inspection throughput. 
In the case of the fixed position monitoring device, a different type of CCD is warranted. In this deployment scenario, a larger format system is needed in order to observe as many fuel assemblies as possible using a single device. Using the previous three pixel resolution calculation, it was determined that a $16 \times 16$ matrix of fuel assemblies could be detected with a $1024 \times 1024$ format system. In this application, there is no need for fast readout of the system, and therefore a full frame, slow scan architecture could be implemented. The slow scan technology reduces the readout noise to about 5 electrons (at $50 \mathrm{kHz}$ ). TE-forced air cooling is still preferred, resulting in a dark current of around 1 electron/pixel-second.

In the fixed position scenario, it would also be possible to account for the interference from the system lighting by performing a baseline calibration and digitally subtracting the background. (This is not practical with the portable system since the viewing angle relative to the illumination changes as the system is moved.) Accounting for the facility illumination through digital background correction means that the system can now be operated over a broader spectral range ( $350-650$ nanometers) where Cerenkov is most readily transmitted through water. It also avoids the use of ultraviolet optical systems which have more absorption, lower quantum efficiencies, and are more expensive to procure.

The system performance of the fixed position detection system is evaluated using the same relationships as the portable monitoring systems. The readout noise is now 5 electrons and the quantum efficiency is 0.30 for a front-illuminated system. Since there is no 10 second throughput requirement for the fixed position monitor, the integration time is limited only by saturation considerations at high signal flux rates (1000 photons/secondpixel) and dark current noise which can be significant for long integration times. The saturation limit can be estimated as follows,

$\mathrm{T}_{\mathrm{SAT}}=\frac{\text { Well Capacity }}{\text { Photon Flux X QE }}=\frac{350,000}{1000 \times 0.30}=1200$

Using a 1200 second integration time and the following relationships,

$\mathrm{N}_{\mathrm{R}}=5$ electrons

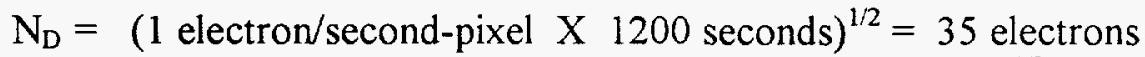

$\mathrm{N}_{\mathrm{ph}}=(5 \text { photons/second-pixel } \mathrm{X} 0.30 \times 1200 \text { seconds })^{1 / 2}=42$ electrons

$\mathrm{N}_{\mathrm{T}}=\left[5^{2}+35^{2}+42^{2}\right]^{1 / 2}=55$ electrons

$\mathrm{S} / \mathrm{N}=\frac{\text { Integrated Photon Flux }}{\text { Noise }\left(\mathrm{N}_{\mathrm{T}}\right)}=\frac{1800}{55}=32.7 @ 3.1 \%$ precision 
The detectability of the system is

Detectability $=\frac{\text { Noise }}{\mathrm{QE}}=\frac{55}{0.30}=183$ photons

Dynamic Range $=\underline{350000}=6363$ or around 12 bits

55

In this case, going to a shorter integration time would actually improve performance due to the relatively high dark current noise. Evaluating the system performance at an integration time of 600 seconds, the following result is obtained,

$N_{R}=5$ electrons

$N_{D}=(1 \text { electron/second-pixel } X 600 \text { seconds })^{1 / 2}=25$ electrons

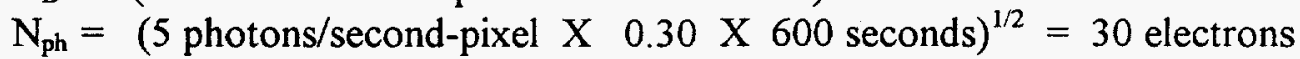

$\mathrm{N}_{\mathrm{T}}=\left[5^{2}+25^{2}+30^{2}\right]^{1 / 2}=39$ electrons

$\mathrm{S} / \mathrm{N}=\frac{\text { Integrated Photon Flux }}{\text { Noise }\left(\mathrm{N}_{\mathrm{T}}\right)}=\frac{900}{39}=23.1 @ 2.6 \%$ precision

The detectability of the system is

Detectability $=\frac{\text { Noise }}{\mathrm{QE}}=\frac{39}{0.30}=130$ photons

Dynamic Range $=\underline{350000}=8974$ or around 13 bits

39

Again, the performance of this system may be limited in its ability to unambiguously detect fuel assemblies in all storage configurations. Also, since this system would operate unattended, a scheme for verifying the integrity of the data would need to be devised. 


\section{CONCLUSIONS}

The design features of CCD systems for both portable and fixed position Cerenkov monitoring scenarios have been developed and are summarized in Table 1. The portable device must have the ability for on-site inspectors to view the fuel at near real-time rates, and in addition, allow detailed data collection from fuel assemblies at a rate of 10 seconds per assembly. These requirements dictate that a frame transfer CCD architecture be used and the integration time for a particular view be limited. In addition, the system requires a direct provision for excluding signal contributions from the facility lighting. This restricts the spectral range of the device to wavelengths shorter than 350 nanometers. Portability of the system requires that TE-forced air cooling be employed, rather than forced water or liquid nitrogen-cooling. While the system performance is adequate for most inspection activities, the fast frame transfer increases the system noise (due to higher readout noise) and the operation at ultraviolet wavelengths reduces the quantum efficiency of the system, resulting in lower precision measurements.

The fixed position Cerenkov detector requires a larger format camera system in order to maximize the number of stored fuel assemblies that can be continuously monitored. In this case, a full frame slow scan architecture is used. Since there are no fast data throughput requirements for this system, the signal integration time is limited only by dark current noise considerations and system saturation at high flux rates. In this scenario, it is feasible to use digital background correction to eliminate signal contributions from facility lighting. This allows the operation of the monitor over a broader spectral range where Cerenkov radiation is most readily transmitted through water. This design also avoids the use of ultraviolet optical components which have more absorption, lower quantum efficiencies and are more expensive to procure. The disadvantage of this deployment scenario is with its political sensitivity and need to verify the integrity of the data.

Both of these system designs may suffer from limited resolution for some storage configurations. In the portable design, the resolution is limited in the interest of maintaining as wide field-of-view as possible in order to increase the number of fuel elements simultaneously detected. Examining many fuel assemblies at once allows a long signal integration time to be used, while maintaining a 10 second per assembly throughput. In the case of the fixed system, the resolution was limited in order to view as many fuel assemblies as possible with a single instrument. The resolution of both devices could be increased by going to larger, more expensive pixel formats. Each system would also benefit from use of a back illuminated CCD chip. This configuration would greatly increase the quantum efficiency; and consequently, allow smaller integration times to be used. The smaller integration time would reduce the considerable dark current noise contribution that is unavoidable with the limited $\left(-40^{\circ} \mathrm{C}\right)$ cooling available with the forced air TE system. 


\begin{tabular}{|c|c|c|c|}
\hline & PORTABLE DEVICE & FIXED DEVICE & COMMENTS \\
\hline Format & $\begin{array}{c}512 \times 512 \\
(20 \mu \mathrm{m} \times 20 \mu \mathrm{m})\end{array}$ & $\begin{array}{c}1024 \times 1024 \\
(20 \mu \mathrm{m} \times 20 \mu \mathrm{m})\end{array}$ & \\
\hline Architecture & Frame Transfer & Slow Scan & \\
\hline View Height & $12 \mathrm{~m}$ & $25 \mathrm{~m}$ & \\
\hline Lens & $75 \mathrm{~mm}$ & $150 \mathrm{~mm}$ & \\
\hline Quantum Efficiency & 0.15 & 0.30 & $\begin{array}{c}\text { Front Illumination } \\
\text { UV Coating } \\
\end{array}$ \\
\hline Integration Time & $600 \mathrm{sec}$ & $600 \mathrm{sec}$ & \\
\hline Readout Noise & $20 \mathrm{e}^{-}$ & $5 \mathrm{e}^{-}$ & \\
\hline Dark Current & 1 e\%/sec-pixel & 1 e/sec-pixel & $\begin{array}{l}-40^{\circ} \mathrm{C} \text { TE Forced Air } \\
\text { Cooling }\end{array}$ \\
\hline Well Capacity & $200,000 \mathrm{e}^{-}$ & $350,000 \mathrm{e}^{-}$ & \\
\hline Detectability & 253 & 130 & \\
\hline Precision & $8.5 \%$ & $2.6 \%$ & \\
\hline Dynamic Range & 5263 (12 bit) & 8974 (13 bit) & \\
\hline
\end{tabular}

Table 1. Portable and Fixed Position Cerenkov Performance Summary 


\section{ACKNOWLEDGMENTS}

This work was supported by the U.S. Department of Energy, Office of Nonproliferation and National Security, under DOE Idaho Operations Office Contract DE-AC0794ID13223.

\section{REFERENCES}

1. N. Nichelson and E.J. Dowdy, "Irradiated Fuel Monitoring by Cerenkov Glow Intensity Measurements," Los Alamos National Laboratory Report LA-8767-MS, March 1981.

2. A. Nilsson, N. Danielson, J.D. Chen, C.J. Young, P. Vodraska, and A. Nakaoka, "Cerenkov Light Images of Irradiated Fuel Assemblies," Proceedings of the $30^{\text {th }}$ Annual Meeting of the Institute of Nuclear Material Management, Vol. XVIII, Orlando, Florida, July 1989, p. 902.

3. E. J. Dowdy, N. Nichelson and J.T. Calwell, "Method for Monitoring Irradiated Fuel Using Cerenkov Radiation," Patent Number 4,389,568, June 1983.

4. "Charge-Coupled Devices for Quantitative Electronic Imaging", Photometrics Technical Pamphlet, Photometrics Ltd., Tucson, AZ, 1992.

5. J. Skalyo, Jr., "Cerenkov Techniques for LWR spent Fuel Observations," Proceedings of the $28^{\text {th }}$ Meeting of the Institute of Nuclear Materials Management, Newport Beach, CA, July 1987.

6. "High Performance Digital CCD Cameras", Princeton Instruments Vendor Literature, Princeton Instruments, Inc., Trenton, NJ, September 1995.

7. K. Kulka and A. Hallgren, "Determination of Detected Cerenkov-Light Intensities for Verification of Irradiated Nuclear Fuel Using Monte Carlo Techniques," SKI Report 95:45, August 1995. 